Moroccan J. of Pure and Appl. Anal. (MJPAA)

Volume 3(1), 2017, Pages 116-132

ISSN: Online 2351-8227 - Print 2605-6364

DOI 10.1515/mjpaa-2017-0010

\title{
Fractional parabolic problems with a nonlocal initial condition
}

\author{
B. Abdellaoui ${ }^{*, 1, a}$, A. Boucherif ${ }^{2, b}$, T.M. Touaoula ${ }^{1, c}$
}

ABSTRACT. In this work we will consider a class of non local parabolic problems with nonlocal initial condition, more precisely we deal with the problem

$$
\left\{\begin{aligned}
u_{t}+(-\Delta)^{s} u & =a(x) u^{p}+g(x, t) & & \text { in } \Omega_{T} \equiv \Omega \times(0, T), \\
u(x, t) & =0 & & \text { in } \mathbb{R}^{n} \backslash \Omega \times(0, T), \\
u(x, 0) & =\int_{0}^{T} u^{\theta}(x, \sigma) d \sigma & & \text { for } x \in \Omega, \\
u(x, t) & \geq 0 & & \text { in } \Omega_{T},
\end{aligned}\right.
$$

where $\Omega$ is a bounded domain, the function $a$ can be a singular potential, $g$ is a suitable function which will be specified later and $p, \theta$ are positive constants. Our main goal is to discuss the existence of a positive solution to the above problem under natural conditions on the data. The question of global existence is also analyzed. 2010 Mathematics Subject Classification. 35B25, 35K58, 35B33, 47G20.

Key words and phrases. Fractional Laplacian parabolic problem, nonlocal initial condition, Hardy potential.

Received January 10, 2018 - Accepted March 25, 2018.

(C)The Author(s) 2017. This article is published with open access by Sidi Mohamed Ben Abdallah University.

* is partially supported by research grants MTM2013-40846-P and MTM2016-80474-P, MINECO, Spain.

1 Laboratoire d'Analyse Nonlinéaire et Mathématiques Appliquées. Département de Mathématiques, Université Abou Bakr Belkaïd, Tlemcen, 13000, Algeria.

2 Department of Mathematics and Statistics. King Fahd University of Petroleum and Minerals. Box 5046 Dhahran, 31261 Saudi Arabia.

a e-mail: boumediene.abdellaoui@inv.uam.es

${ }^{b}$ e-mail: boucherif@yahoo.com

${ }^{c}$ e-mail: touaoula_tarik@yahoo.fr 


\section{Introduction}

In this paper we discuss the existence of positive solutions of the following nonlocal parabolic problem subject to an initial condition of integral type

$$
\left\{\begin{aligned}
u_{t}+(-\Delta)^{s} u & =a(x) u^{p}+g(x, t) & & \text { in } \Omega_{T} \equiv \Omega \times(0, T), \\
u(x, t) & =0 & & \text { in } \mathbb{R}^{n} \backslash \Omega \times(0, T), \\
u(x, 0) & =\int_{0}^{T} u^{\theta}(x, \sigma) d \sigma & & \text { for } x \in \Omega, \\
u(x, t) & \geq 0 & & \text { in } \Omega_{T},
\end{aligned}\right.
$$

where $\Omega$ is a bounded domain in $\mathbb{R}^{N}$ and $(-\Delta)^{s}$ is the fractional Laplacian of order $2 s, s \in(0,1)$, defined by

$$
(-\Delta)^{s} u(x):=a_{N, s} \text { P.V. } \int_{\mathbb{R}^{N}} \frac{u(x)-u(y)}{|x-y|^{N+2 s}} d y
$$

with

$$
a_{N, s}=2^{2 s-1} \pi^{-\frac{N}{2}} \frac{\Gamma\left(\frac{N+2 s}{2}\right)}{|\Gamma(-s)|}
$$

is a normalization constant.

Our main goal is to introduce natural conditions on $a, g, p, \theta$ in order to get the existence of a positive solution to $(1)$.

Fractional diffusion equations are abstract partial differential equations that involve fractional derivatives in space and/or time. They are useful to model anomalous diffusion, where a plume of particles spreads in a different manner than the classical diffusion equation predicts. See for instance [4].

Parabolic problems (case $s=1$ ) with initial or boundary conditions of integral type appear in the modeling of concrete problems, such as heat conduction [8], [17], [9], thermoelasticity [11]. Several papers have been devoted to the study of parabolic problems with integral conditions. We refer to [6], [7], [13], [16] [10], [20], [21] and the references therein. A good account on numerical treatment of parabolic problems with integral conditions can be found in $[12]$.

For $s<1$, the study of parabolic problem with the fractional Laplacian has received more attention in the past years. We refer for example to [18] where existence results are proved including for $L^{1}$ data. We shall analyze the fractional parabolic problem with a class of nonlinearities of the form $a(x) u^{p}+g$ where $a$ can be a singular potential, and the nonlocal initial condition $u(x, 0)=\int_{0}^{T} u^{\theta}(x, s) d s$. This initial condition seems to be new in the case of fractional diffusion problems. The paper is organized as follows. In section two we present some tools from functional analysis, like fractional Sobolev spaces, the definitions of energy and weak solutions, the comparison principle and the weak Harnack inequality that are useful to complete our study. Section three is devoted to the case $a(x)=1, g(x, t)=0$. Under suitable hypotheses on $p$ and $\theta$ we are able to show the existence of a non trivial solution. The question of global existence or blow up in finite time is studied in subsection 3.1. In the last Section we consider the singular case $a(x)=\frac{\lambda}{|x|^{2 s}}$. This case is related to the well known Hardy inequality. The main objective of this section is to establish the existence of a critical exponent $\theta^{*}$ such that for $\theta<\theta^{*}$ our problem has a solution. We also prove a nonexistence result. As a consequence, we get a complete blow up for a family of approximating problems.

\section{Functional setting and preliminaries results.}

In this section we will provide the precise functional setting that is necessary for the study of fractional diffusion problems, also we shall state and prove some preliminary results.

Let $\Omega \subset \mathbb{R}^{N}$ and assume that $s \in(0,1)$. The fractional Sobolev space $H^{s}(\Omega)$ is defined by

$$
H^{s}(\Omega) \equiv\left\{u \in L^{2}(\Omega): \int_{\Omega} \int_{\Omega} \frac{|u(x)-u(y)|^{2}}{|x-y|^{N+2 s}} d x d y<+\infty\right\} .
$$


$H^{s}(\Omega)$ is a Banach space endowed with the following norm

$$
\|u\|_{H^{s}(\Omega)}=\left(\int_{\Omega}|u(x)|^{2} d x\right)^{\frac{1}{2}}+\left(\int_{\Omega} \int_{\Omega} \frac{|u(x)-u(y)|^{2}}{|x-y|^{N+2 s}} d x d y\right)^{\frac{1}{2}} .
$$

Now, we define the space

$$
H_{0}^{s}(\Omega)=\left\{u \in H^{s}\left(\mathbb{R}^{N}\right) \text { such that } u=0 \text { a.e. in } \mathbb{R}^{N} \backslash \Omega\right\} .
$$

If $\Omega$ is a bounded domain then using a Poincaré type inequality we can endowed $H_{0}^{s}(\Omega)$ with the norm

$$
\|u\|_{H_{0}^{s}(\Omega)}=\left(\iint_{D_{\Omega}} \frac{|u(x)-u(y)|^{2}}{|x-y|^{N+2 s}} d x d y\right)^{1 / 2},
$$

where $D_{\Omega}=\mathbb{R}^{N} \times \mathbb{R}^{N} \backslash(\mathcal{C} \Omega \times \mathcal{C} \Omega)$. We refer to [14] for more properties of the fractional Sobolev spaces. Notice that

$$
(-\Delta)^{s}: H_{0}^{s}(\Omega) \rightarrow H^{-s}(\Omega)
$$

is a continuous operator.

It is clear that, for all $u, v \in H_{0}^{s}(\Omega)$,

$$
2 a_{N, s}^{-1} \int_{\mathbb{R}^{N}} u(-\Delta)^{s} v d x=\iint_{D_{\Omega}} \frac{(u(x)-u(y))(v(x)-v(y))}{|x-y|^{N+2 s}} d x d y .
$$

We define now the space $L^{2}\left(0, T ; H_{0}^{s}(\Omega)\right)$ as the set of function $u$ such that $u \in L^{2}\left(\Omega_{T}\right)$ and $\|u\|_{L^{2}\left(0, T ; H_{0}^{s}(\Omega)\right)}<\infty$ where

$$
\|u\|_{L^{2}\left(0, T ; H_{0}^{s}(\Omega)\right)}=\left(\int_{0}^{T} \iint_{D_{\Omega}} \frac{|u(x, t)-u(y, t)|^{2}}{|x-y|^{N+2 s}} d x d y d t\right)^{\frac{1}{2}} .
$$

$L^{2}\left(0, T ; H_{0}^{s}(\Omega)\right)$ equipped with the above norm is a Banach spaces.

Let us begin by precising the sense in which solutions to problem (1) are defined.

Consider the problem

$$
\left\{\begin{aligned}
u_{t}+(-\Delta)^{s} u & =f(x, t) & & \text { in } \Omega \times(0, T), \\
u(x, t) & =0 & & \text { in }\left(\mathbb{R}^{N} \backslash \Omega\right) \times(0, T), \\
u(x, 0) & =u_{0}(x) & & \text { if } x \in \Omega .
\end{aligned}\right.
$$

If $\left(f, u_{0}\right) \in L^{2}\left(\Omega_{T}\right) \times L^{2}(\Omega)$, we can deal with energy solution, more precisely we have the next definition.

Definition 2.1. Assume $\left(f, u_{0}\right) \in L^{2}\left(\Omega_{T}\right) \times L^{2}(\Omega)$. We say that $u$ is an energy solution to problem $(3)$ if $u \in$ $L^{2}\left(0, T ; H_{0}^{s}(\Omega)\right) \cap \mathcal{C}\left([0, T], L^{2}(\Omega)\right), u_{t} \in L^{2}\left(0, T ; H^{-s}(\Omega)\right)$ and for all $v \in L^{2}\left(0, T ; H_{0}^{s}(\Omega)\right)$ we have

$$
\begin{aligned}
& \int_{0}^{T}\left\langle u_{t}, v\right\rangle d t+\frac{1}{2} \int_{0}^{T} \iint_{D_{\Omega}} \frac{(u(x, t)-u(y, t))(v(x, t)-v(y, t)}{|x-y|^{N+2 s}} d x d y d t \\
& =\iint_{\Omega_{T}} f(x, t) v(x, t) d x d t
\end{aligned}
$$

and $u(x,.) \rightarrow u_{0}$ strongly in $L^{2}(\Omega)$ as $t \rightarrow 0$.

Notice that the existence and uniqueness of an energy solution to the problem (3) can be proved by means of a direct Hilbert space approach. We refer to [2] for more details.

As a consequence we get the next comparison principle.

Lemma 2.2. (Weak Comparison Principle). Assume that $u, v \in L^{2}\left(0, T ; H_{0}^{s}(\Omega)\right) \cap \mathcal{C}\left([0, T], L^{2}(\Omega)\right)$ are such that

$$
\left\{\begin{array} { l } 
{ u _ { t } + ( - \Delta ) ^ { s } u = f _ { 1 } \text { in } \Omega \times ( 0 , T ) , } \\
{ u = h _ { 1 } \text { in } ( \mathbb { R } ^ { N } \backslash \Omega ) \times ( 0 , T ) , } \\
{ u ( x , 0 ) = u _ { 0 } ( x ) \text { in } \Omega , }
\end{array} \quad \left\{\begin{array}{l}
v_{t}+(-\Delta)^{s} v=f_{2} \text { in } \Omega \times(0, T), \\
v=h_{2} \text { in }\left(\mathbb{R}^{N} \backslash \Omega\right) \times(0, T), \\
v(x, 0)=v_{0}(x) \text { in } \Omega .
\end{array}\right.\right.
$$

Then, if $f_{1} \leq f_{2}, h_{1} \leq h_{2}$ and $u_{0} \leq v_{0}$, then $u \leq v$ in $\mathbb{R}^{N} \times(0, T)$. 
Since, some times we need to pass to the limit in a very weak sense, then we need to define the sense weak. Define the set

$$
\begin{gathered}
\mathcal{T}:=\left\{\phi: \mathbb{R}^{N} \times[0, T] \rightarrow \mathbb{R}, \text { s.t. }-\phi_{t}+(-\Delta)^{s} \phi=\varphi, \varphi \in L^{\infty}(\Omega \times(0, T)),\right. \\
\left.\phi=0 \text { in }\left(\mathbb{R}^{N} \backslash \Omega\right) \times(0, T], \phi(x, T)=0 \text { in } \Omega\right\} .
\end{gathered}
$$

Notice that every $\phi \in \mathcal{T}$ belongs in particular to $L^{\infty}(\Omega \times(0, T))$ (see [18]).

Definition 2.3. Assume that $\left(f, u_{0}\right) \in L^{1}\left(\Omega_{T}\right) \times L^{1}(\Omega)$. We say that $u \in \mathcal{C}\left([0, T) ; L^{1}(\Omega)\right)$ is a weak supersolution (subsolution) of problem (3) if $u=0$ in $\left(\mathbb{R}^{N} \backslash \Omega\right) \times(0, T)$ and for all nonnegative $\phi \in \mathcal{T}$ we have that

$$
\int_{0}^{T} \int_{\Omega}-\phi_{t} u d x d t+\int_{0}^{T} \int_{\Omega} u(-\Delta)^{s} \phi d x d t \geq(\leq) \int_{0}^{T} \int_{\Omega} f \phi d x d t+\int_{\Omega} u_{0}(x) \phi(x, 0) d x .
$$

If $u$ is super and subsolution, then we say that $u$ is a weak solution.

In the case where the data are non negative we have a weak version of the well known Harnack inequality whose proof can be found in [15].

Theorem 2.4. Assume that $f, u_{0} \supsetneqq 0$, if $u$ is a non negative supersolution of $(3)$ in $\Omega \times(0, T)$, then there exist $r>0$ and a positive constant $C=C\left(N, s, r, t_{0}, \beta\right)$ such that

$$
\iint_{R^{-}} u(x, t) d x d t \leq C\left(\text { essinf } \operatorname{Rin}_{R^{+}} u\right)
$$

where $R^{-}=B_{r}(0) \times\left(t_{0}-\frac{3}{4} \beta, t_{0}-\frac{1}{4} \beta\right), R^{+}=B_{r}(0) \times\left(t_{0}+\frac{1}{4} \beta, t_{0}+\frac{3}{4} \beta\right)$.

\section{Existence result: regular case}

In this section we will assume that $a(x)=1$ and $g(x, t)=0$. It is clear that in this situation $u=0$ is a solution to $(1)$.

Of course, we are interested in nontrivial solutions. In fact, we shall show that under appropriate conditions on $p$ and $\theta$, we can prove the existence of a nontrivial solution. Our first existence result in this direction is the following.

Theorem 3.1. Assume that $0<p<1 \leq \theta$, then the problem (1) has a nontrivial positive solution.

Proof. We will use a monotone argument. Let $v(t)=((1-p) t)^{\frac{1}{1-p}}$, then, $v$ solves

$$
v^{\prime}(t)=v^{p}(t), v(0)=0 .
$$

Since $p<1$, we define $w$ to be the unique positive solution to the problem

$$
\left\{\begin{aligned}
(-\Delta)^{s} w & =w^{p} & & \text { in } \Omega \\
w & =0 & & \text { in } \mathbb{R}^{n} \backslash \Omega,
\end{aligned}\right.
$$

Notice that the existence of $w$ follows using classical minimizing argument, however the uniqueness follows from [1] and [18]. It is not difficult to show also that $w \in L^{\infty}(\Omega)$.

We claim that there exists $T_{0}>0$ such that $w$ is a super-solution to (1) in $\Omega_{T}$ for all $T \leq T_{0}$.

Indeed, since $w \in L^{\infty}(\Omega)$ and $\theta \geq 1$, then $w(x) \geq c(\Omega) w^{\theta}(x)$ for all $x \in \Omega$. Thus we get the existence of $T_{0}>0$ such that $w(x) \geq T_{0} w^{\theta}(x)$ for all $x \in \Omega$. Consequently $w$ is a super-solution to problem $(1)$ in $\Omega \times(0, T)$ with $T \leq T_{0}$.

Define now $z(x, t)=v(\varepsilon t) w(x)$, then for $(x, t) \in \Omega_{T}$ we have

$$
\begin{aligned}
z_{t}+(-\Delta)^{s} z & =\varepsilon v^{\prime}(\varepsilon t) w+v(\varepsilon t) w^{p} \\
& =\varepsilon v^{p}(\varepsilon t) w+v(\varepsilon t) w^{p}, \\
& \leq v^{p}(\varepsilon t) w^{p}=z^{p}(x, t)
\end{aligned}
$$


for $\varepsilon$ so small. On the other hand,

$$
0=z(x, 0) \leq \int_{0}^{T} z^{\theta}(x, \sigma) d \sigma .
$$

Thus $z$ is a sub-solution to problem (1) with $z(x, t) \leq w(x)$ in $\Omega_{T}$ at least for $T$ small.

We define now the sequence $u_{n}$ by $u_{1}=z$ and $u_{n+1}$ solves

$$
\left\{\begin{aligned}
\left(u_{n+1}\right)_{t}+(-\Delta)^{s} u_{n+1} & =u_{n}^{p} & & \text { in } \Omega_{T}, \\
u_{n+1}(x, t) & =0 & & \text { in }\left(\mathbb{R}^{n} \backslash \Omega\right) \times(0, T), \\
u_{n+1}(x, 0) & =\int_{0}^{T} u_{n}^{\theta}(x, \sigma) d \sigma & & \text { for } x \in \Omega .
\end{aligned}\right.
$$

It is clear that $u_{n}$ is increasing in $n$ and $u_{n} \in L^{\infty}\left(\Omega_{T}\right), u_{n} \in L^{2}\left(0, T, H_{0}^{s}(\Omega)\right)$ and $z \leq u_{n} \leq w$ for all $n$.

Using the Dominated Convergence Theorem, we obtain that $u_{n} \rightarrow \tilde{u}$ in $L^{2}\left(\Omega_{T}\right), \tilde{u} \in L^{2}\left(0, T ; H_{0}^{s}(\Omega)\right)$ and $\tilde{u}=0$ in $\mathbb{R}^{N} \backslash \Omega \times(0, T)$. Since $u_{n} \leq w \in L^{\infty}\left(Q_{T}\right)$, then we can prove that $\left\{u_{n}\right\}_{n}$ is bounded in $\mathcal{C}\left([0, T], L^{\alpha}(\Omega)\right)$ for all $\alpha>0$. Thus, using the fact that $\left\{u_{n}\right\}_{n}$ is increasing, it follows that

$$
u_{n}(x, 0) \rightarrow \tilde{u}(x, 0),
$$

strongly in $L^{\alpha}(\Omega)$ for all $\alpha>0$, in particular for, $\alpha=\theta$. Thus $\tilde{u}$ solves the problem (1) with $\tilde{u} \neq 0$.

Let us consider now the case $\theta<1$.

Theorem 3.2. Assume that $0<p<1$ and $\theta<1$, then there exists $T_{0}>0$ such that the problem (1) has at least a non trivial positive weak solution $\tilde{u}$ in $\Omega_{T}$. In addition, if $\theta \leq \frac{1}{2}$, then the solution $\tilde{u} \in L^{2}\left(0, T ; H_{0}^{s}(\Omega)\right)$.

Proof. It is clear that $z$ defined in the previous theorem is a sub-solution. Since $w=0$ in $\left(\mathbb{R}^{n} \backslash \Omega\right) \times(0, T)$, using the fact that $\theta<1$ we see that $w$ is not a supersolution to problem (1) at leat near the boundary of $\Omega$. To avoid this difficulty we will prove a direct apriori estimate on the sequence $\left\{u_{n}\right\}_{n}$ defined as in the previous proof. It is clear that $u_{n} \leq u_{n+1}$.

We claim that $\left\|u_{n}\right\|_{L^{1}\left(Q_{T}\right)} \leq C$ for all $n$. Define

$$
\Psi_{n}(x, t)=\int_{0}^{t} u_{n}(x, \sigma) d \sigma,
$$

then $\Psi_{n}(x, 0)=0$ and $\left(\Psi_{n}\right)_{t}(x, t)=u_{n}(x, t)$. Thus

$$
\left(\Psi_{n}\right)_{t}+(-\Delta)^{s} \Psi_{n}=\int_{0}^{T} u_{n-1}^{\theta}(x, \sigma) d \sigma+\int_{0}^{t} u_{n-1}^{p}(x, \sigma) d \sigma .
$$

Since $p<1$, then using the fact that $u_{n-1} \leq u_{n}$ and Young and Hölder inequalities, it holds

$$
\left(\Psi_{n}\right)_{t}+(-\Delta)^{s} \Psi_{n} \leq t^{1-p} \Psi_{n}^{p}+\int_{0}^{T} u_{n}(x, \sigma) d \sigma+c(T, \theta) .
$$

In what follows we denote by $C$ any positive constant depending only on the data and independent of $u_{n}$.

Define $\varphi$ to be the unique solution to problem

$$
\left\{\begin{array}{rll}
(-\Delta)^{s} \varphi & =1 & \text { in } \Omega, \\
\varphi & =0 & \text { in } \mathbb{R}^{n} \backslash \Omega, \\
\varphi & \geq 0 & \text { in } \Omega,
\end{array}\right.
$$

it is clear that $\varphi \in L^{\infty}(\Omega)$. Using $\varphi$ as a test function in (6), it follows that

$$
\frac{\partial}{\partial t} \int_{\Omega} \Psi_{n}(x, t) \varphi d x+\int_{\Omega} \Psi_{n}(x, t) d x \leq t^{1-p} \int_{\Omega} \Psi_{n}^{p}(x, t) \varphi(x) d x+\int_{0}^{T} \int_{\Omega} u_{n}(x, \sigma) \varphi(x) d x d \sigma+C .
$$

Let $Y_{n}(t)=\int_{\Omega} \Psi_{n}(x, t) \varphi(x) d x$, then $Y_{n}^{\prime}(t) \geq 0$. Therefore using Young inequality,

$$
Y_{n}^{\prime}(t) \leq \varepsilon t^{1-p} Y_{n}(t)+\int_{0}^{T} Y_{n}(\sigma) d s+C(\varepsilon)
$$


Integrating in $(0, T)$ and using the fact that $Y_{n}^{\prime}(t) \geq 0$, we get

$$
Y_{n}(T) \leq \varepsilon T^{2-p} Y_{n}(T)+T \int_{0}^{T} Y_{n}(\sigma) d \sigma+\bar{C}(T, \varepsilon) .
$$

Thus it holds that

$$
\left(1-\varepsilon T^{2-p}-T^{2}\right) Y_{n}(T) \leq C(T, \varepsilon) .
$$

Choosing $T>0$ such that $\left(1-\varepsilon T^{2-p}-T^{2}\right) \geq \frac{1}{2}$, then we conclude that $Y_{n}(T) \leq \tilde{C}(\varepsilon, T)$. Hence,

$$
\int_{0}^{T} \int_{\Omega} \Psi_{n}(x, t) \varphi(x) d x d t \leq C .
$$

In view of (8) we reach

$$
\int_{0}^{T} \int_{\Omega} \Psi_{n}(x, t) d x d t \leq C_{1}
$$

thus

and consequently,

$$
\left\|u_{n}\right\|_{L^{1}\left(Q_{T}\right)} \leq C_{1}
$$

$$
\left\{\begin{array}{l}
\left\|u_{n}^{p}\right\|_{L^{1}\left(Q_{T}\right)} \leq C, \\
\left\|u_{n}(x, 0)\right\|_{L^{1}(\Omega)} \leq C,
\end{array}\right.
$$

for all $n$. Therefore we get the existence of $\tilde{u}$ such that $u_{n} \uparrow \tilde{u}$ strongly in $L^{1}\left(Q_{T}\right)$. It is not difficult to show that $\tilde{u}$ solves (1) at least in the weak sense.

Assume now that $\theta \leq \frac{1}{2}$, using $u_{n+1}$ as a test function in problem (5), there results that

$$
\begin{aligned}
& \frac{1}{2} \int_{\Omega} u_{n+1}^{2}(x, T) d x+\frac{1}{2} \int_{0}^{T} \iint_{D_{\Omega}} \frac{\left|u_{n+1}(x, \sigma)-u_{n+1}(y, \sigma)\right|^{2}}{|x-y|^{N+2 s}} d x d y d \sigma \\
\leq & \int_{0}^{T} \int_{\Omega} u_{n+1}^{p+1}(x, s) d x d s+\frac{1}{2} \int_{\Omega} u_{n+1}^{2}(x, 0) d x \\
\leq & \int_{0}^{T} \int_{\Omega} u_{n+1}^{p+1}(x, s) d x d s+\frac{1}{2} \int_{\Omega}\left(\int_{0}^{T} u_{n+1}^{\theta}(x, s) d s\right)^{2} d x .
\end{aligned}
$$

Since $p<1$ and $\theta \leq \frac{1}{2}$, then using Hölder and Young inequalities we obtain

$$
\begin{aligned}
& \frac{1}{2} \int_{\Omega} u_{n+1}^{2}(x, T) d x+\frac{1}{2} \int_{0}^{T} \iint_{D_{\Omega}} \frac{\left|u_{n+1}(x, \sigma)-u_{n+1}(y, \sigma)\right|^{2}}{|x-y|^{N+2 s}} d x d y d \sigma \\
\leq & \int_{0}^{T} \int_{\Omega} u_{n+1}^{2}(x, s) d x d s+C(\Omega, T) \int_{0}^{T} \int_{\Omega} u_{n+1}(x, t) d x d t+C(\Omega, T) .
\end{aligned}
$$

It is clear that following the same computations as above, we can prove that for all $t<T$, we have

$$
\begin{aligned}
& \frac{1}{2} \int_{\Omega} u_{n+1}^{2}(x, t) d x+\frac{1}{2} \int_{0}^{t} \iint_{D_{\Omega}} \frac{\left|u_{n+1}(x, \sigma)-u_{n+1}(y, \sigma)\right|^{2}}{|x-y|^{N+2 s}} d x d y d \sigma \\
\leq & \int_{0}^{t} \int_{\Omega} u_{n+1}^{2}(x, s) d x d s+C(\Omega, T) \int_{0}^{T} \int_{\Omega} u_{n+1}(x, t) d x d t+C(\Omega, T) .
\end{aligned}
$$

Now, taking into consideration that $\left\|u_{n}\right\|_{L^{1}\left(Q_{T}\right)} \leq C_{1}$ and using Gronwall inequality we conclude that $\left\|u_{n}\right\|_{L^{2}\left(0, T ; H_{0}^{s}(\Omega)\right)} \leq$ $C_{2}$. Thus $\tilde{u} \in L^{2}\left(0, T ; H_{0}^{s}(\Omega)\right)$ and then $\tilde{u}$ is an energy solution to problem (1).

\section{Remark 3.1.}

(1) The same existence result holds if we consider problem (1) with $0<p<1<\theta$ and $0 \supsetneqq g$ be such that $\sup _{t \in(0, T)} g(., t)=f_{T} \in L^{m}(\Omega)$ with $m>\frac{N}{2 s}$. To see that we have just to built a suitable supersolution. Define $w_{T}$ to be the unique positive solution to problem

$$
\left\{\begin{aligned}
(-\Delta)^{s} w_{T} & =w_{T}^{p}+f_{T} & & \text { in } \Omega, \\
w_{T} & =0 & & \text { in } \mathbb{R}^{n} \backslash \Omega .
\end{aligned}\right.
$$


Taking into consideration the regularity of $f_{T}$, we get $w \in L^{\infty}(\Omega)$. Now, choosing $T$ such that $T w^{\theta-1} \leq 1$, it holds that $w_{T}$ is a supersolution to problem (1). Hence the existence result follows using the previous iteration schema.

(2) In the case where $\max \{p, \theta\}<1$, we can prove that problem (1) has at least a distributional solution for all $0 \supsetneqq g \in L^{1}\left(\Omega_{T}\right)$ and for all $T>0$. To see that, we fix $T>0$ and define the sequence $\left\{u_{n}\right\}_{n}$ by setting $u_{1}=0$ and $u_{n+1}$ is the unique solution to problem

$$
\left\{\begin{aligned}
\left(u_{n+1}\right)_{t}+(-\Delta)^{s} u_{n+1} & =u_{n}^{p}+g_{n} & & \text { in } \Omega_{T}, \\
u_{n+1}(x, t) & =0 & & \text { in }\left(\mathbb{R}^{n} \backslash \Omega\right) \times(0, T), \\
u_{n+1}(x, 0) & =\int_{0}^{T} u_{n}^{\theta}(x, \sigma) d \sigma & & \text { for } x \in \Omega .
\end{aligned}\right.
$$

where $g_{n}=\min \{g, n\}$. It is clear that $u_{n}$ is well defined, $u_{n} \in L^{\infty}\left(\Omega_{T}\right), u_{n} \in L^{2}\left(0, T, H_{0}^{s}(\Omega)\right)$ and $0 \supsetneqq u_{n} \leq u_{n+1}$ for all $n$.

Let $\phi_{1} \in C^{0, \alpha}(\bar{\Omega})$ be the unique positive solution to problem

$$
\left\{\begin{aligned}
(-\Delta)^{s} \phi_{1} & =\lambda_{1} \phi & & \text { in } \Omega \\
\phi_{1} & =0 & & \text { in } \mathbb{R}^{n} \backslash \Omega, \\
\left\|\phi_{1}\right\|_{L^{1}} & =1 . & &
\end{aligned}\right.
$$

Using $\phi_{1}$ as a test function in (9) and taking into consideration the monotony of $u_{n}$, it follows that

$$
\frac{\partial}{\partial t} \int_{\Omega} u_{n}(x, t) \phi_{1}(x) d x+\lambda_{1} \int_{\Omega} u_{n}(x, t) \phi_{1}(x) d x \leq \int_{\Omega} u_{n}^{p}(x, t) \phi_{1} d x+\int_{\Omega} g_{n}(x, t) \phi_{1} d x .
$$

Thus,

$$
\begin{gathered}
\int_{\Omega} u_{n}(x, T) \phi_{1}(x) d x+\lambda_{1} \int_{0}^{T} \int_{\Omega} u_{n}(x, \sigma) \phi_{1}(x) d x \\
\leq \int_{0}^{T} \int_{\Omega} u_{n}^{p}(x, \sigma) \phi_{1} d x+\int_{0}^{T} \int_{\Omega} u_{n}^{\theta}(x, \sigma) \phi_{1} d x+\left\|\phi_{1}\right\|_{L^{\infty}(\Omega)}\|g\|_{L^{1}\left(\Omega_{T}\right)} .
\end{gathered}
$$

Since $\max \{p, \theta\}<1$, then using Young inequality it holds that

$$
\int_{\Omega} u_{n}(x, T) \phi_{1}(x) d x+C_{1} \int_{0}^{T} \int_{\Omega} u_{n}(x, \sigma) \phi_{1}(x) d x \leq C_{2} T\left\|\phi_{1}\right\|_{L^{1}(\Omega)}+C_{2} .
$$

Let $\varphi$ be the unique solution to problem (7), it is clear that $\varphi \simeq \phi_{1}$. Using $\varphi$ as a test function in (9) and taking into consideration the above estimates, it holds that $\left\|u_{n}\right\|_{L^{1}\left(Q_{T}\right)} \leq C_{1}$. Thus for all $n$,

$$
\left\{\begin{array}{l}
\left\|u_{n}^{p}\right\|_{L^{1}\left(Q_{T}\right)} \leq C \\
\left\|u_{n}(x, 0)\right\|_{L^{1}(\Omega)} \leq C .
\end{array}\right.
$$

Hence we get the existence of $\tilde{u}$ such that $u_{n} \uparrow \tilde{u}$ strongly in $\mathcal{C}\left([0, T], L^{1}(\Omega)\right)$ and in $\left.L^{(} Q_{T}\right)$. It is not difficult to show that $\tilde{u}$ solves (1) at least in the weak sense.

We deal now with the case $p>1$. For a classical initial condition, if $u(x, 0)=0$, using Gronwall's inequality we can prove that the unique bounded nonnegative solution to (1) (with $g \equiv 0$ ) is $u \equiv 0$. For a nonlocal initial condition, the situation seems to be different.

Theorem 3.3. Assume that $0<\theta<1<p<2_{s}^{*}-1$ and $g \equiv 0$, then there exists $T_{0}>0$ such that the problem (1) has at least a non trivial positive weak solution $\tilde{u}$ in $\Omega_{T}$.

Proof. Since $p<2_{s}^{*}-1$, we define $\bar{w}$ to be a positive solution to the problem

$$
\left\{\begin{array}{rll}
(-\Delta)^{s} \bar{w}=\bar{w}^{p} & \text { in } \Omega \\
\bar{w}=0 & \text { in } \mathbb{R}^{n} \backslash \Omega \\
\bar{w} \geq 0 & \text { in } \Omega
\end{array}\right.
$$


The existence of $\bar{w}$ follows using classical mountain pass theorem, in the same way we get easily that $w \in L^{\infty}(\Omega) \cap$ $\mathcal{C}^{0, \alpha}(\Omega)$. Choosing $T>\|w\|_{L^{\infty}(\Omega)}^{1-\theta}$, it holds that $w$ is a subsolution to problem (1) with $g \equiv 0$.

Fix $T$ as above and define the sequence $\left\{u_{n}\right\}_{n}$ by setting $u_{1}=\bar{w}$ and $u_{n+1}$ is the unique bounded solution to problem

$$
\left\{\begin{aligned}
\left(u_{n+1}\right)_{t}+(-\Delta)^{s} u_{n+1} & =u_{n+1}^{p} & & \text { in } \Omega_{T}, \\
u_{n+1}(x, t) & =0 & & \text { in }\left(\mathbb{R}^{n} \backslash \Omega\right) \times(0, T), \\
u_{n+1}(x, 0) & =\int_{0}^{T} u_{n}^{\theta}(x, \sigma) d \sigma & & \text { for } x \in \Omega .
\end{aligned}\right.
$$

Notice that the existence and the regularity of $u_{n+1}$ follows using classical semi-group theory. It is clear that for $n$ fixed $u_{n} \in L^{\infty}\left(\Omega_{T}\right) \cap L^{2}\left(0, T, H_{0}^{s}(\Omega)\right)$ and $0 \supsetneqq u_{n} \leq u_{n+1}$ for all $n$.

Fix the set $A \equiv B_{r} \times\left(t_{1}, t_{2}\right) \subset \subset \Omega_{T}$ and define $v$ to be the unique solution to problem

$$
\left\{\begin{aligned}
-v_{t}+(-\Delta)^{s} v & =\chi_{A} & & \text { in } \Omega_{T}, \\
v(x, t) & =0 & & \text { in }\left(\mathbb{R}^{n} \backslash \Omega\right) \times(0, T), \\
v(x, T) & =0 & & \text { for } x \in \Omega .
\end{aligned}\right.
$$

Using $v$ as a test function in (12) and integrating in $\Omega_{T}$, we get

$$
\int_{t_{1}}^{t_{2}} \int_{B_{r}} u_{n+1} d x d t=\int_{0}^{T} \int_{\Omega} u_{n+1}^{p} v d x d t+\int_{\Omega} u_{n+1}(x, 0 v(x, 0) d x d t .
$$

Thus

$$
\int_{0}^{T} \int_{\Omega} u_{n+1}^{p} v d x d t \leq \int_{t_{1}}^{t_{2}} \int_{B_{r}} u_{n+1} d x d t
$$

Since $p>1$, taking into consideration that $v \geq C$ in $B_{r} \times\left(t_{1}, t_{2}\right)$ and using Young inequality it follows that

$$
\int_{0}^{T} \int_{\Omega} u_{n+1}^{p} v d x d t \leq C \text { for all } n
$$

Define now $v_{1}$ to be the unique solution to problem

$$
\left\{\begin{array}{rlll}
-v_{1 t}+(-\Delta)^{s} v_{1} & =1 & & \text { in } \Omega_{T} \\
v_{1}(x, t) & =0 & & \text { in }\left(\mathbb{R}^{n} \backslash \Omega\right) \times(0, T), \\
v_{1}(x, T) & =0 & & \text { for } x \in \Omega .
\end{array}\right.
$$

Using $v_{1}$ as a test function in (12), using the fact that $v \simeq v_{1}$ and by estimate (14), we reach that $\left\|u_{n}\right\|_{L^{1}\left(Q_{T}\right)} \leq C_{1}$. Hence the monotony of the sequence $\left\{u_{n}\right\}_{n}$ and the above argument allow us to get the existence of a distributional solution $u$ such that $u_{n} \uparrow \tilde{u}$ strongly in $\left.L^{(} Q_{T}\right)$ and $u^{p} \in L_{l o c}^{1}\left(Q_{T}\right)$.

Remark 3.2. It is clear that the hypothesis $\theta<1$ allows us to built a suitable supersolution and to pass to the limit in the initial data once proving the strong convergence in $L^{1}\left(Q_{T}\right)$. This argument does not work if $\theta>1$ which seems to be more complicated.

3.1. Existence and non existence of global solution. In this subsection we analyze the question of global existence of positive solution to problem (1). We will prove that the exponent $\theta$ plays a crucial role in this regard. Before starting our analysis we need to precise the sense of global solution.

Definition 3.4. Assume that $g: \Omega \times \mathbb{R} \rightarrow \mathbb{R}^{+}$is a continuous function. We say that problem (1) has a global solution if there exists a function $u$ defined in $\Omega \times(0, \infty)$ such that $u^{p} \in L^{1}(\Omega \times(0, T))$ for all $T>0, \int_{0}^{\infty} \int_{\Omega} u^{\theta}(x, \sigma) d x d \sigma<\infty$ and $u$ solves $(1)$ in $\Omega \times(0, \infty)$ at least in the sense of distribution.

Let us begin by the next existence result. 
Theorem 3.5. Assume that $\min \{p, \theta\}>1, a(x)=1$ and $g(x, t)=A e^{-\alpha t}$ where $\alpha<\lambda_{1}$ the first eigenvalue and $A$ is a small constant depending only on the data. Suppose that $\theta>1$, then the problem

$$
\left\{\begin{aligned}
u_{t}+(-\Delta)^{s} u & =u^{p}+A e^{-\alpha t} & & \text { in } \Omega \times(0, \infty), \\
u(x, t) & =0 & & \text { in } \mathbb{R}^{n} \backslash \Omega \times(0, \infty), \\
u(x, 0) & =\int_{0}^{\infty} u^{\theta}(x, \sigma) d \sigma & & \text { for } x \in \Omega,
\end{aligned}\right.
$$

has a minimal global bounded solution $u$ such that $u \in L^{q}(\Omega \times(0, \infty)$ for all $q \geq 1$.

Proof. We begin by the construction of a suitable supersolution. Fix $\alpha<\lambda_{1}$ and consider $\varphi$, the minimal solution to the problem

$$
\left\{\begin{aligned}
(-\Delta)^{s} \varphi-\alpha \varphi & =\varphi^{p}+c_{0} & & \text { in } \Omega \\
\varphi & =0 & & \text { in } \mathbb{R}^{n} \backslash \Omega, \\
\varphi & \geq 0 & & \text { in } \Omega .
\end{aligned}\right.
$$

Notice that the existence of a bounded regular minimal solution $\varphi$ follows using classical monotone argument when $c_{0}$ is small.

Now define $\bar{u}(x, t)=A e^{-\alpha t} \varphi$. we claim that $\bar{u}$ is a supersolution to problem (16). We have

$$
\begin{aligned}
\bar{u}_{t}+(-\Delta)^{s} \bar{u} & =A e^{-\alpha t}\left((-\Delta)^{s} \varphi-\alpha \varphi\right) \\
& =A e^{-\alpha t} \varphi^{p}+c_{0} A e^{-\alpha t} \\
& \geq \bar{u}^{p}+c_{0} A e^{-\alpha t} .
\end{aligned}
$$

On the other hand, taking into consideration that $\theta>1$ and choosing $A$ small, it holds

$$
\bar{u}(x, 0)=A \varphi(x) \geq \frac{A^{\theta} \varphi^{\theta}}{\theta \alpha}=\int_{0}^{\infty} \bar{u}^{\theta}(x, \sigma) d \sigma .
$$

Thus the claim follows.

It is clear that $\underline{u} \equiv 0$ is a strict subsolution.

Now, define the sequence $\left\{u_{n}\right\}_{n}$ with $u_{0}=0$ and $u_{n+1}$ solves

$$
\left\{\begin{aligned}
\left(u_{n+1}\right)_{t}+(-\Delta)^{s} u_{n+1} & =u_{n}^{p}+c_{0} A e^{-\alpha t} & & \text { in } \Omega \times(0, \infty), \\
u_{n+1}(x, t) & =0 & & \text { in } \mathbb{R}^{n} \backslash \Omega \times(0, \infty), \\
u_{n+1}(x, 0) & =\int_{0}^{\infty} u_{n}^{\theta}(x, \sigma) d \sigma & & \text { for } x \in \Omega .
\end{aligned}\right.
$$

It is clear that $u_{n}$ is increasing in $n, u_{n} \leq \bar{u}$ for all $n$ and that $\left\{u_{n}\right\}_{n}$ is bounded in $L^{\infty}(\Omega \times(0, \infty)) \cap L^{2}\left(0, \infty, H_{0}^{s}(\Omega)\right)$. Using the monotone convergence Theorem and using $u_{n+1}$ as a test function in (17) we conclude that $u_{n} \uparrow u$ strongly in $L^{q}\left(\Omega \times(0, \infty)\right.$ for all $q \in[1, \infty], u \in L^{2}\left((0, \infty), H_{0}^{s}(\Omega)\right)$ and $u$ solves $(16)$.

\section{Remark 3.3.}

(1) Using comparison arguments we can prove the existence of global solution if $g$ satisfies $g(x, t) \leq A e^{-\alpha t}$ with $\alpha<\lambda$ and $A$ small.

(2) In the case of "a natural initial" condition, finite blow up holds if $\left\|u_{0}\right\|_{L^{1}(\Omega)}$ is large, see for instance [5]. This makes a significant difference with respect to our case.

In the case where $\theta \leq 1$ and $p>1$, then under suitable condition on $\Omega$ we can prove a non existence of global solution. More precisely we have

Theorem 3.6. Suppose that $\theta \leq 1, p>1$ and $g \geq 0$. If $\lambda_{1}(\Omega)<1$, problem (1) has a non global solution.

Proof. Without loss of generality we can assume that $g=0$.

Let us begin by the case $\theta=1$. We argue by contradiction. Assume that $u$ is a nontrivial global solution to problem (1). Let $\phi_{1} \in C^{0, \alpha}(\bar{\Omega})$ be the unique positive solution to problem (10). Define $v(x, t)=\int_{0}^{t} u(x, s) d s$, then, 
$v(x, 0)=0$ and $v(., \infty)=u_{0}(.) \in L^{1}(\Omega)$. It is clear that

$$
v_{t}+(-\Delta)^{s} v=\int_{0}^{\infty} u(x, \sigma) d \sigma+\int_{0}^{t} u^{p}(x, \sigma) d \sigma
$$

Using Hölder inequality it holds

$$
v_{t}+(-\Delta)^{s} v \geq v(x, \infty)+\frac{1}{t^{\frac{p}{p^{\prime}}}} v^{p}
$$

It is clear that $v(x, t)=0$ in $\mathbb{R}^{n} \backslash \Omega \times(0, T)$, thus using $\phi_{1}$ as a test function in (18), we reach that

$$
\begin{aligned}
\frac{\partial}{\partial t} \int_{\Omega} v(x, t) \phi_{1}(x) d x+\lambda_{1} \int_{\Omega} v(x, t) \phi_{1}(x) d x & \geq \int_{\Omega} v(x, \infty) \phi_{1} d x+\frac{1}{t^{\frac{p}{p^{\prime}}}} \int_{\Omega} v^{p}(x, t) \phi_{1}(x) d x \\
& \geq \int_{\Omega} v(x, \infty) \phi_{1} d x+\frac{1}{t^{\frac{p}{p^{\prime}}}}\left(\int_{\Omega} v(x, t) \phi_{1}(x) d x\right)^{p}
\end{aligned}
$$

Let $y(t)=\int_{\Omega} v(x, t) \phi_{1}(x) d x$, then $y^{\prime} \geq 0$ and $y(t) \rightarrow y(\infty)=\int_{\Omega} v(x, \infty) \phi_{1}(x) d x=\int_{\Omega} u_{0}(x) \phi_{1}(x) d x$. Thus $y^{\prime}(t) \rightarrow 0$ as $t \rightarrow \infty$. From (19), we obtain that

$$
y^{\prime}(t)+l_{1} y(t) \geq y(\infty)+\frac{1}{t^{\frac{p}{p^{\prime}}}} y^{p}(t) .
$$

Letting $t \rightarrow \infty$, we reach that $l_{1} \geq 1$ a contradiction with the main hypothesis. Hence we conclude in this case.

We deal now with the case $\theta<1$. As above setting $v(x, t)=\int_{0}^{t} u(x, s) d s$, it follows that $v(x, 0)=0$ and

$$
v_{t}+(-\Delta)^{s} v=\int_{0}^{\infty} u^{\theta}(x, \sigma) d \sigma+\int_{0}^{t} u^{p}(x, \sigma) d \sigma \geq \int_{0}^{t}\left(u^{\theta}(x, \sigma)+u^{p}(x, \sigma)\right) d \sigma
$$

Since $\theta<1<p$, then for $x \geq 0$, then we obtain that

$$
x^{\theta}+x^{p} \geq C_{1} x+C_{2} x^{p}
$$

Therefore we reach that

$$
v_{t}+(-\Delta)^{s} v \geq C_{1} v+C_{2} \frac{1}{t^{\frac{p}{p^{\prime}}}} v^{p} .
$$

Now the rest of the proof follows exactly as in the first case $\theta=1$.

\section{Existence and non existence result: singular case}

In this section we will assume $0 \in \Omega$ and $a(x)=\frac{\lambda}{|x|^{2 s}}$ and $p=1$. Then problem (1) takes the form

$$
\left\{\begin{aligned}
u_{t}+(-\Delta)^{s} u & =l \frac{u}{|x|^{2 s}} & & \text { in } \Omega_{T} \equiv \Omega \times(0, T), \\
u(x, t) & =0 & & \text { in } \mathbb{R}^{n} \backslash \Omega \times(0, T), \\
u(x, 0) & =\int_{0}^{T} u^{\theta}(x, \sigma) d \sigma & & \text { for } x \in \Omega, \\
u(x, t) & \geq 0 & & \text { in } \Omega_{T},
\end{aligned}\right.
$$

This problem is related to the following Hardy inequality;

Theorem 4.1. (Fractional Hardy inequality). Assume that $\phi \in \mathcal{C}_{0}^{\infty}\left(\mathbb{R}^{N}\right)$, then

$$
\frac{a_{N, s}}{2} \iint_{\mathbb{R}^{N} \times \mathbb{R}^{N}} \frac{|\phi(x)-\phi(y)|^{2}}{|x-y|^{N+2 s}} d x d y \geq \Lambda_{N, s} \int_{\mathbb{R}^{N}} \frac{\phi^{2}}{|x|^{2 s}} d x,
$$

where

$$
\Lambda_{N, s}=2^{2 s} \frac{\Gamma^{2}\left(\frac{N+2 s}{4}\right)}{\Gamma^{2}\left(\frac{N-2 s}{4}\right)} .
$$


The constant $\Lambda_{N, s}$ is optimal and is not attained. Moreover, $\Lambda_{N, s} \rightarrow \Lambda_{N, 1}:=\left(\frac{N-2}{2}\right)^{2}$, the classical Hardy constant, when $s \rightarrow 1$. If $\Omega$ is bounded domain such that $0 \in \Omega$, then (21) takes the form

$$
\frac{a_{N, s}}{2} \iint_{D_{\Omega}} \frac{|\phi(x)-\phi(y)|^{2}}{|x-y|^{N+2 s}} d x d y \geq \Lambda_{N, s} \int_{\Omega} \frac{\phi^{2}}{|x|^{2 s}} d x, \phi \in H_{0}^{s}(\Omega)
$$

with the same constant $\Lambda_{N, s}$.

Define

$$
\gamma=\frac{N-2 s}{2}-\alpha,
$$

where

$$
\lambda=\lambda(\alpha)=\lambda(-\alpha)=\frac{2^{2 s} \Gamma\left(\frac{N+2 s+2 \alpha}{4}\right) \Gamma\left(\frac{N+2 s-2 \alpha}{4}\right)}{\Gamma\left(\frac{N-2 s+2 \alpha}{4}\right) \Gamma\left(\frac{N-2 s-2 \alpha}{4}\right)} .
$$

Then $w(x)=|x|^{-\gamma} \in H^{s}(\Omega)$ solves the equation

$$
(-\Delta)^{s} w=\lambda \frac{w}{|x|^{2 s}} \text { in } \mathbb{R}^{N}
$$

From [2], we know that if $u$ is a positive supersolution to the equation

$$
u_{t}+(-\Delta)^{s} u=\lambda \frac{u}{|x|^{2 s}} \text { in } \Omega \times(0, T),
$$

then for all $B_{r}(0) \times\left(t_{1}, t_{2}\right) \subset Q_{T}$, there exists a positive constant $C$ independents of $u$ such that $u(x, t) \geq C|x|^{-\gamma}$ in $B_{r}(0) \times\left(t_{1}, t_{2}\right)$.

The next result proved in [2] will be essential to our analysis.

Theorem 4.2. Define

Consider the problem

$$
p_{+}(\lambda, s)=1+\frac{2 s}{\frac{N-2 s}{2}-\alpha}=1+\frac{2 s}{\gamma}
$$

$$
\left\{\begin{aligned}
u_{t}+(-\Delta)^{s} u & =l \frac{u}{|x|^{2 s}}+u^{p} & & \text { in } \Omega \times(0, T), \\
u(x, t) & =0 & & \text { in }\left(\mathbb{R}^{N} \backslash \Omega\right) \times[0, T), \\
u(x, 0) & =u_{0}(x) \geqslant 0 & & \text { if } x \in \Omega .
\end{aligned}\right.
$$

If $p>p_{+}(\lambda, s)$, then problem (25) has non distributional supersolution in any domain of the form $B_{r}(0) \times(0, T)$.

We are now able to state the next non existence result.

Theorem 4.3. Assume that $\theta>p_{+}(\lambda, s)$, then problem (20) has no positive weak solution.

Proof. We argue by contradiction. Assume that $u$ is a weak positive solution to (20) with $\theta>p_{+}(\lambda, s)$. Fix $B_{r}(0) \times\left(0, t_{1}\right) \subset Q_{T}$, then $u \in L^{1}\left(B_{r}(0) \times\left(0, t_{1}\right)\right), \frac{u}{|x|^{2 s}} \in L^{1}\left(B_{r}(0) \times\left(0, t_{1}\right)\right)$ and $u_{0} \in L^{1}\left(B_{r}(0)\right)$.

For $(x, t) \in B_{r}(0) \times\left(0, t_{1}\right)$ we define $v(x, t)=\int_{0}^{t} u(x, \sigma) d \sigma$, then $v$ solves

$$
v_{t}+(-\Delta)^{s} v=\lambda \frac{v}{|x|^{2 s}}+u(x, 0) .
$$

Taking into consideration that $\theta>1$, it holds

$$
\begin{aligned}
v_{t}+(-\Delta)^{s} v & \geq \lambda \frac{v}{|x|^{2 s}}+\int_{0}^{t_{1}} u^{\theta}(x, \sigma) \\
& \geq \lambda \frac{v}{|x|^{2 s}}+C\left(t_{1}\right) v^{\theta}
\end{aligned}
$$

Recall that $\theta>p_{+}(\lambda, s)$, then $v$ is a nontrivial supersolution to $(25)$ in $B_{r}(0) \times\left(0, t_{1}\right) \subset Q_{T}$, a contradiction with the non existence result of Theorem (4.2). Hence we conclude. 
As a consequence we get the next complete blow-up result.

Theorem 4.4. Assume that $g \in L^{\infty}\left(\Omega_{T}\right)$ is such that $g \supsetneqq 0$ in $\Omega_{T}$. Let $u_{n}$ be the unique a solution to the problem

$$
\left\{\begin{aligned}
u_{n t}+(-\Delta)^{s} u_{n} & =\lambda \frac{u_{n-1}}{|x|^{2 s}+\frac{1}{n}}+g(x, t) & & \text { in } \Omega \times(0, T), \\
u_{n}(x, t) & =0 & & \text { in }\left(\mathbb{R}^{N} \backslash \Omega\right) \times[0, T), \\
u_{n}(x, 0) & =\int_{0}^{T} u_{n-1}^{\theta}(x, s) d s & & \text { if } x \in \Omega,
\end{aligned}\right.
$$

with $u_{0}=0$ and $\theta>p_{+}(\lambda, s)$. Then $u_{n}\left(x_{0}, t_{0}\right) \rightarrow \infty$, for all $\left(x_{0}, t_{0}\right) \in \Omega \times(0, T)$.

Proof. Notice that the existence of $u_{n}$ follows using classical result for the fractional diffusion equation. We claim that $\left\{u_{n}\right\}_{n}$ is an increasing sequence in $n$. We will apply the Comparison Principle, it is clear that $u_{1} \geq u_{0} \equiv 0$. Define $v=u_{2}-u_{1}$, it is clear that

$$
\left\{\begin{aligned}
v_{t}+(-\Delta)^{s} v & =\lambda \frac{v}{|x|^{2 s}+\frac{1}{n}} & & \text { in } \Omega \times(0, T), \\
v(x, t) & =0 & & \text { in }\left(\mathbb{R}^{N} \backslash \Omega\right) \times[0, T), \\
v(x, 0) & =\int_{0}^{T}\left(u_{1}^{\theta}(x, s)-u_{0}^{\theta}(x, s)\right) d s \geq 0 & & \text { in } \in \Omega,
\end{aligned}\right.
$$

thus $v \geq 0$ and then $u_{2} \geq u_{1}$. Therefore an induction argument allows us a show that $u_{n} \geq u_{n-1}$ and the claim follows.

The proof of the blow up result follows closely using the argument of [2]. For the reader convenience we include here the details.

Suppose, by contradiction, that there exists $\left(x_{0}, t_{0}\right) \in \Omega \times(0, T)$ such that

$$
u_{n}\left(x_{0}, t_{0}\right) \rightarrow C_{0}<\infty \text { as } n \rightarrow \infty \text {. }
$$

Using the weak Harnack inequality stated in Theorem 2.4, we get the existence of $r_{0}>0$ and a positive constant $C=C\left(N, s_{0}, t_{0}\right)$ such that

$$
\iint_{R_{0}^{-}} u_{n}(x, t) d x d t \leq C \underset{R_{0}^{+}}{\operatorname{essinf}} u_{n} \leq C,
$$

where $R_{0}^{-}=B_{r_{0}}\left(x_{0}\right) \times\left(t_{0}-\frac{3}{4} \beta, t_{0}-\frac{1}{4} \beta\right)$ and $R_{0}^{+}=B_{r_{0}}\left(x_{0}\right) \times\left(t_{0}+\frac{1}{4} \beta, t_{0}+\frac{3}{4} \beta\right)$.

Without loss of generality, we can suppose $x_{0}=0$. If not, we can use the same iteration argument as in [2], namely we can consider a sequence of points $\left\{x_{i}\right\}_{i=0}^{k}$ with $x_{k}=0$, and a sequence of radius $\left\{r_{i}\right\}_{i=0}^{k}$ such that $B_{r_{i}}\left(x_{i}\right) \subset \Omega, B_{r_{i}}\left(x_{i}\right) \cap B_{r_{i+1}}\left(x_{i+1}\right) \neq \emptyset$, for all $i=0, \ldots, k$ and, by the Hanarck inequality,

$$
\iint_{R_{i}^{-}} u_{n}(x, t) d x d t \leq C \underset{R_{i}^{+}}{\operatorname{ess} \inf } u_{n},
$$

where $R_{i}^{-}=B_{r_{i}}\left(x_{i}\right) \times\left(t_{i}-\frac{3}{4} \beta, t_{i}-\frac{1}{4} \beta\right)$ and $R_{i}^{+}=B_{r_{i}}\left(x_{i}\right) \times\left(t_{i}+\frac{1}{4} \beta, t_{i}+\frac{3}{4} \beta\right), t_{i} \in(0, T)$ and $\beta$ is small enough so that $t_{i}-\frac{3}{4} \beta>0$ and $t_{i}+\frac{3}{4} \beta<T$ for all $i=0, \ldots, k$. Now, we chose $t_{i}=t_{i-1}-\beta$ for $i=1, \ldots, k$, son we obtain that

$$
\left(t_{i}+\frac{1}{4} \beta, t_{i}+\frac{3}{4} \beta\right)=\left(t_{i-1}-\frac{3}{4} \beta, t_{i-1}-\frac{1}{4} \beta\right)
$$

and then $R_{i}^{+} \cap R_{i-1}^{-} \neq \emptyset$. Therefore using again Harnack inequality and taking into consideration the above construction, we conclude that

$$
\begin{aligned}
\iint_{R_{i}^{-}} u_{n}(x, t) d x d t & \leq \underset{R_{i}^{+}}{\operatorname{essinf}} u_{n}(x, t) \leq \operatorname{ess} \inf _{R_{i}^{+} \cap R_{i-1}^{-}} u_{n}(x, t) \\
& \leq C \iint_{R_{i-1}^{-}} u_{n}(x, t) d x d t \\
& \leq \ldots \leq C \iint_{R_{0}^{-}} u_{n}(x, t) d x d t \leq \tilde{C}
\end{aligned}
$$


Hence iterating the above process we reach that

$$
\iint_{R_{k}^{-}} u_{n}(x, t) d x d t \leq C \text { for all } n
$$

with $R_{k}^{-}=B_{r_{k}}(0) \times\left(t_{k}-\frac{3}{4} \beta, t_{k}-\frac{1}{4} \beta\right)$. For simplicity we write $B_{r_{k}}(0) \times\left(t_{k}-\frac{3}{4} \beta, t_{k}-\frac{1}{4} \beta\right)=B_{r}(0) \times\left(t_{1}, t_{2}\right)$.

Now using the monotony of $\left\{u_{n}\right\}_{n}$, we get the existence of a measurable function $u$ such that $u_{n} \uparrow u$ strongly in $L^{1}\left(B_{r}(0) \times\left(t_{1}, t_{2}\right)\right)$.

We claim that

$$
\left\|\frac{u_{n-1}}{|x|^{2 s}}\right\|_{L^{1}\left(B_{r}(0) \times\left(t_{1}, t_{2}\right)\right)}+\left\|u_{n-1}^{\theta}\right\|_{L^{1}\left(B_{r}(0) \times(0, T)\right)} \leq C \text { for all } n .
$$

To see that, we define $\varphi$ as the unique solution to the problem

$$
\left\{\begin{aligned}
-\varphi_{t}+(-\Delta)^{s} \varphi & =\chi_{B_{r}(0) \times\left[t_{1}, t_{2}\right]} \text { in } \Omega \times(0, T) \\
\varphi(x, t) & >0 \text { in } \Omega \times(0, T) \\
\varphi(x, t) & =0 \text { in }\left(\mathbb{R}^{N} \backslash \Omega\right) \times[0, T) \\
\varphi(x, T) & =0 \text { in } \Omega
\end{aligned}\right.
$$

Using $\varphi$ as a test function in (26), it follows that

$$
\begin{gathered}
\iint_{\Omega_{T}} u_{n}\left(-\varphi_{t}+(-\Delta)^{s} \varphi\right) d x d t \\
=\lambda \iint_{\Omega_{T}} \frac{u_{n-1} \varphi}{|x|^{2 s}+\frac{1}{n}} d x d t+\int_{\Omega} \varphi(x, 0) \int_{0}^{T} u_{n-1}^{\theta}(x, s) d s d x .
\end{gathered}
$$

Taking into consideration estimate (27),

$$
\iint_{\Omega_{T}} u_{n}\left(-\varphi_{t}+(-\Delta)^{s} \varphi\right) d x d t=\iint_{\left.B_{r}(0) \times\left(t_{1}, t_{2}\right)\right)} u_{n}(x, t) d x d t \leq C .
$$

Now using the fact that $\varphi(x, 0) \geq C$ in $B_{r}(0)$, we conclude that

$$
\lambda \iint_{\left.B_{r}(0) \times\left(t_{1}, t_{2}\right)\right)} \frac{u_{n-1}}{|x|^{2 s}+\frac{1}{n}} d x d t+\int_{B_{r}(0)} \int_{0}^{T} u_{n-1}^{\theta}(x, s) d s d x \leq C
$$

and then the claim follows.

We define now the new sequence $\left\{v_{n}\right\}_{n}$ by $v_{0}=0$ and $v_{n}$ solves

$$
\left\{\begin{aligned}
v_{n t}+(-\Delta)^{s} v_{n} & \left.=\lambda \frac{v_{n-1}}{|x|^{2 s}+\frac{1}{n}}+g(x, t) \text { in } B_{r}(0) \times\left(t_{1}, t_{2}\right)\right), \\
v_{n}(x, t) & =0 \operatorname{in}\left(\mathbb{R}^{N} \backslash B_{r}(0)\right) \times\left(t_{1}, t_{2}\right) \\
v_{n}\left(x, t_{1}\right) & =\int_{t_{1}}^{t_{2}} v_{n-1}^{\theta}(x, s) d s \text { if } x \in B_{r}(0)
\end{aligned}\right.
$$

As above we can prove that the sequence $\left\{v_{n}\right\}_{n}$ is increasing in $n$ and that $v_{n} \leq u_{n}$ in $\left.B_{r}(0) \times\left(t_{1}, t_{2}\right)\right)$ for all $n$. Thus

$$
\lambda \iint_{\left.B_{r}(0) \times\left(t_{1}, t_{2}\right)\right)} \frac{v_{n-1}}{|x|^{2 s}+\frac{1}{n}} d x d t+\int_{B_{r}(0)} \int_{t_{1}}^{t_{2}} v_{n-1}^{\theta}(x, s) d s d x \leq C
$$

and then we can prove that $v_{n} \uparrow v$ that solves $(25)$, in $\left.B_{r}(0) \times\left(t_{1}, t_{2}\right)\right)$ at least in a weak sense. This is a contradiction with the non existence result of Theorem 4.2 .

In the case where $\theta \leq 1$, we can prove the following existence result.

Theorem 4.5. Assume that $\theta \leq 1$ and $\lambda<\Lambda_{N, s}$. Suppose in addition that $0 \supsetneqq g(x, t) \leq \frac{C}{|x|^{\gamma+2 s}}$ for a suitable positive constant $C$. Then then problem (20) has a minimal positive solution. 
Proof. We have just to built a suitable supersolution. Fix $\lambda_{1} \in\left(\lambda, \Lambda_{N, s}\right)$ and define $w_{1}(x)=|x|^{-\gamma}$ where $\gamma$ is defined in (23) where $\lambda$ is replaced by $\lambda_{1}$. Recall that $w_{1} \in H^{s}(\Omega)$ and then $w_{1} \in L^{2}(\Omega)$.

We claim that $w_{1}$ is a supersolution to problem (20) in $\Omega_{T}$ for some $T>0$. Notice that by definition we have

$$
\left(w_{1}\right)_{t}+(-\Delta)^{s} w_{1}=\lambda_{1} \frac{w_{1}}{|x|^{2 s}}=\lambda \frac{w_{1}}{|x|^{2 s}}+\frac{\left(\lambda_{1}-\lambda\right)}{|x|^{\gamma_{1}+2 s}} .
$$

Hence choosing $C \leq\left(\lambda_{1}-\lambda\right)$, it follows that

$$
\left(w_{1}\right)_{t}+(-\Delta)^{s} w_{1} \geq \lambda \frac{w_{1}}{|x|^{2 s}}+g(x, t) .
$$

On the other hand, using the fact that $\theta<1$, then for all $x \in \Omega$, we have

$$
w_{1}(x) \geq C(\Omega) w_{1}^{\theta}(x) \geq \int_{0}^{T} w_{1}^{\theta}(x),
$$

for any $T \leq C(\Omega)$. Fix $T$ as above, then $w$ is a supersolution to (20) and the claim follows.

We define now the sequence $u_{n}$ by $u_{1}=0$ and $u_{n+1}$ solves

$$
\left\{\begin{aligned}
\left(u_{n+1}\right)_{t}+(-\Delta)^{s} u_{n+1} & =\lambda \frac{u_{n}}{|x|^{2 s}+\frac{1}{n}}+g_{n}(x, t) & & \text { in } \Omega_{T}, \\
u_{n+1}(x, t) & =0 & & \text { in } \mathbb{R}^{n} \backslash \Omega \times(0, T), \\
u_{n+1}(x, 0) & =\int_{0}^{T} u_{n}^{\theta}(x, s) d s & & \text { for } x \in \Omega,
\end{aligned}\right.
$$

where $g_{n}=\frac{g}{1+\frac{g}{n}}$. It is clear that $u_{n}$ is an increasing in $n, u_{n} \in L^{2}\left(0, T, H_{0}^{s}(\Omega)\right)$ and $u_{n} \leq w_{1}$ for all $n$. Using $u_{n+1}$ as a test function in (29), using Hardy inequality and taking into consideration that $u_{n} \leq w_{1}$, it follows that

$$
\begin{aligned}
& \frac{1}{2} \int_{\Omega} u_{n+1}^{2}(x, t) d x+\frac{1}{2}\left(1-\frac{\lambda}{\Lambda_{N, s}}\right) \int_{0}^{t} \iint_{D_{\Omega}} \frac{\left|u_{n+1}(x, \sigma)-u_{n+1}(y, \sigma)\right|^{2}}{|x-y|^{N+2 s}} d x d y d \sigma \\
\leq & \frac{T^{2}}{2} \int_{\Omega} w_{1}^{2 \theta} d x \leq C(\Omega, T) .
\end{aligned}
$$

Hence $\left\|u_{n}\right\|_{L^{2}\left(0, T ; H_{0}^{s}(\Omega)\right)} \leq C_{2}$. Using the monotonicity of the sequence $\left\{u_{n}\right\}_{n}$ we reach the existence of $u=$ $\lim \sup u_{n}$ such that $u \in L^{2}\left(0, T ; H_{0}^{s}(\Omega)\right)$ and $u$ solves (20). It is clear that if $v$ is an other solution of (20), then an induction argument allows us to conclude that $v \geq u_{n}$ for all $n$. Therefore $u \leq v$ and then we conclude.

Remark 4.1. Define $\theta^{*}=p_{+}(\lambda, s)$, it seems that the critical exponent for the existence is $\theta^{*}$, however the argument of monotony seems to be difficult to apply since, for any supersolution to the equation

$$
u_{t}+(-\Delta)^{s} u \geq \lambda \frac{u}{|x|^{2 s}} \text { in } \Omega_{T}
$$

blows-up near any point of the form $(0, t)$ and then for $\theta>1$, the nonlocal initial condition does not holds.

\section{References}

[1] B. Abdellaoui, R. Benteffour, Caffarelli-Kohn-Nirenbgerg type inequalities of fractional order and applications. Journal of Functional Analysis. 272 (2017), no. 10, 3998-4029.

[2] B. Abdellaoui, M. Medina, I. Peral, A. Primo Optimal results for the fractional heat equation involving the Hardy potential. Nonl. Anal. TMA. 140 (2016), 166-207.

[3] R. A. Adams Sobolev spaces, Academic Press, New York, 1975.

[4] B. Baeumer, S. Kurita, M.M. Meerschaert Inhomogeneous fractional diffusion equations. Fractional Calculus and Applied Analysis 8(4), 371-386.

[5] H. BEI Blow-up theories for semilinear parabolic equations. Lecture notes in mathematics, 2018.

[6] A. Boucherif Nonlocal problems for parabolic inclusions. 7th AIMS Conference, Dynamical systems, differential equations and applications. Discrete Contin. Dyn. Syst. 2009, suppl. 82-91. 
[7] A. Boucherif Semilinear evolution inclusions with nonlocal conditions. Appl. Math. Lett. 22 (2009), no. 8, $1145-1149$.

[8] J. R. CANnon The solution of the heat equation subject to the specification of energy, Quart. Appl. Math. 21 (1963), 155-160.

[9] R. Yu Chegis Numerical solution of a heat conduction problem with an integral boundary condition, Litovsk. Mat. Sb. 24 (1984), 209-215.

[10] D.H. Dai and Yu Huang Remarks on a semilinear heat equation with integral boundary conditions, Nonl. Anal. 67 (2007), 468-475.

[11] W. A. DAY A decreasing property of solutions of parabolic equations with applicatons to thermoelasticity, Quart. Appl. Math. 41 (1983), 468-475.

[12] M. DehGhan Efficient techniques for the second order parabolic equation subject to nonlocal specifications, Appl. Numer. Math. 52 (2005), 39-62.

[13] K. Deng Exponential decay of solutions of semilinear parabolic equations with nonlocal initial conditions, J. Math. Anal. Appl. 179 (1993), 630-637.

[14] E. Di Nezza, G. Palatucci, E. Valdinoci Hitchhiker's guide to the fractional Sobolev spaces, Bull. Sci. math. 136 (2012), no. 5, 521-573.

[15] M. Felsinger, M. Kassmann, Local regularity for parabolic nonlocal operators. Comm. PDE, 38 (2013) $1539-1573$.

[16] A. Gladkov, M. Guedda Blow-up problem for semilinear heat equation with absorption and a nonlocal boundary condition, Nonl. Anal. TMA. 74 (2011), 4573-4580.

[17] N. IONKIN AND E. MoICEEvSolution of boundary value problem in heat conduction theory with nonlocal boundary conditions, Differential Equations (1977), 294-304, 1977.

[18] T.Leonori, I. Peral, A. Primo, F. Soria Basic estimates for solutions of a class of nonlocal elliptic and parabolic equations. Discrete and Contin- uous Dynamical Systems, 35 (2015)

[19] J. L. Lions Quelques methodes de resolution des problemes aux limites nonlineaires Edition Dunod, Paris 1969.

[20] W. E. Olmstead And C. A. Roberts The one dimensional heat equation with a nonlocal initial condition, Appl. Math. Lett. 10 (1997), 89-94.

[21] H-M. Yin On a class of parabolic equations with nonlocal boundary conditions, J. Math. Anal. Appl. 294 (2004), 712-728. 